\title{
A gamma radiation exposure risk assessment using Monte Carlo simulation and an adult anthropomorphic phantom
}

\author{
Youssef El-Ouardi ${ }^{1, *}$, Adil Aknouch ${ }^{2}$, Ahmed Dadouch $^{1}$, Mohammed Mouhib ${ }^{3}$, and Mounir Benmessaoud ${ }^{1}$ \\ ${ }^{1}$ Nuclear Engineering Group, LISTA Laboratory, Department of Physics, Faculty of Science Dhar El Mahraz,University Sidi \\ Mohamed Ben Abdellah, City Fez,Morocco. \\ ${ }^{2}$ Department of Physics, Nuclear Physics and Techniques Team, Faculty of Science, Ibn Tofail University, Kenitra, Morocco \\ ${ }^{3}$ National Institute for Agronomical Research (NIAR), Irradiation Facility of Boukhalef (SIBO), Tangier, Morocco.
}

\begin{abstract}
The human body is vulnerable to exposure to ionizing radiation permanently. These radiations can be of natural origin such as soil and space, or of artificial and medical origins etc. Despite the great benefits that come from the applications of ionizing radiation in human life can turn at any time into threats to the life of the population once there is serious exposure to ionizing radiation. The aim of this work is to assess the risks of exposure by gamma radiation using the Monte Carlo simulation and an anthropomorphic phantom. This study is carried out on gamma rays received from the cobalt-60 irradiator of the National Institute of Agronomic Research (NIAR) Tangier / Morocco. With the code Geant4 we calculate the absorbed dose by the whole phantom body placed in front of the gamma irradiator inside the NIAR ionization cell. Dose calculations are made as a function of three parameters: phantom-irradiator distance, exposure time and as a function of gamma irradiator activity. The results clearly show that the dose absorbed and thus the danger of irradiation decreases by increasing the phantom-source distance, and by reducing the exposure time and the activity of the cobalt- 60 source.
\end{abstract}

\section{Introduction}

The use of ionizing radiation in human life has become essential and continuously increasing. These types of applications are found in several fields, for example in the medical field, ionizing radiation is used for the diagnosis of diseases and the treatment of carcinogenic tumors. Also they are found in industrial, semi-industrial and research fields etc.[1]

Through the routine use of ionizing radiation sources, accidents and nuclear or radiological incidents can occur, due either to a technical problem, an inattention of an operator, or the unconsciousness of victims or others, which can cause exposure to ionizing radiation to the human body. The severity of these exposures depends on several factors such as the types of ionizing radiation involved, exposure time, body-source distance, sensitivity of the target (organs and tissues), the activity of the sources and the age of victim etc.[2,3]

In this work, we make a Monte Carlo simulation with the Geant 4 code of the doses received by the body of a human assimilated a mathematical anthropomorphic phantom placed in front of a gamma irradiator of cobalt60 inside the NIAR ionization cell.

The objective of this work is then to characterize and quantify the dangers of exposure to ionizing radiation which come from the gamma irradiator of NIAR Marocain according to the activity of the source pencils of cobalt-60, exposure time and of the phantom-source distance. All calculations are made where the source rods are in irradiation position (not included in their storage container), further to quantify the effect of distance, we place the phantom in four positions inside the ionization cell; at 1, 1.5, 2 and 2.5m from the center of the irradiator's source cage.

\section{Materials and methods}

\subsection{The NIAR gamma irradiator}

The NIAR was built in 1995 under the supervision of the IAEA, with the objective of developing Moroccan agriculture by improving agricultural production techniques. in the NIAR there is a parallelepipid-shaped ionization cell housing a panoramic gamma irradiator of cobalt-60 containing 8 pencils sources of total activity $26769 \mathrm{Ci}(07 / 10 / 2020), 45 \mathrm{~cm}$ long and $4.48 \mathrm{~mm}$ radius , fixed in a circular distribution of $20 \mathrm{~cm}$ in diameter. Below the source cage there is a lead container with an external radius equal to $43.5 \mathrm{~cm}$ for storing the irradiator during non-working hours. .[4]

\subsection{The Geant4 simulation code and the digital phantom}

Geant4(Geometry And Tracking) is a simulation tool based on the monte carlo method created at CERN in the beginning with FORTRAN language, currently it is

\footnotetext{
*orresponding author: youssef.elouardi1@usmba.ac.ma
} 
designed in object oriented $\mathrm{C}++$ language. Geant 4 is known throughout the world and allows a wide application in several fields: in nuclear medicine, radiotherapy, radiology, with large cyclotrons and accelerators and in space physics, etc. In this work we use the Monte Carlo GEANT4 simulation code with its version 10.5 (December 7, 2018).It is Very flexible and puts in the hands of the user several models allowing him to track particles like gamma and electrons up to low energies $[5,6]$.

To start applying a Monte Carlo simulation code for such situation, it must first be validated. One of most of the methods used for the validation of calculation codes is to make a comparison between the experimental and simulation results, the error between the two results must be less than $10 \%$. So, to validate the Geant 4 code, a comparison was performed between two results, experimentally by measuring the absorbed dose in 8 positions inside the ionization cell using the Fricke dosimetry system, and with Geant 4 by keeping the same conditions of the experiment as much as possible. The Geant 4 code was validated with an error between the two results lower than $9 \%$. for more details see Y. Elouardi et al. [7]

In this work, we model an adult man by a mathematical anthropomorphic model phantom of MIRD type as shown in figure 1. [8-10]

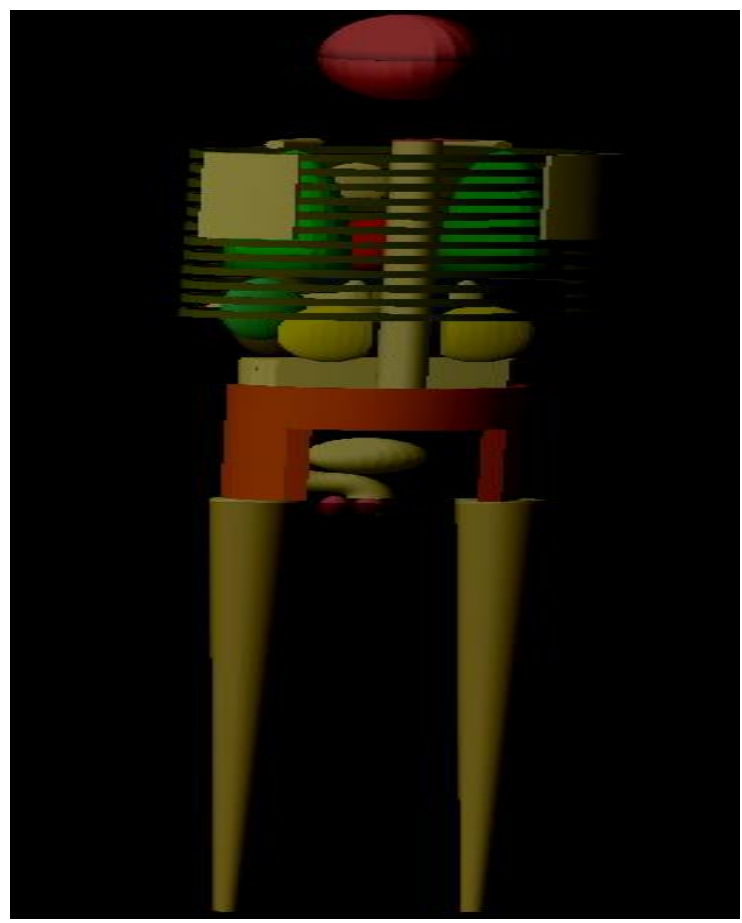

Fig. 1. The anthropomorphic phantom used in this work.

By code Geant 4 , we simulate the dose absorbed by the whole body, then, by using relation (1) we calculate the absorbed dose rate, where $\mathrm{A}$ is the total activity of the source rods, $\mathrm{N}$ is the number of particles generated, the factor 2 is to take into account that at each disintegration of cobalt- 60 there is emission of two gamma particles with respectively equal energies of 1173 and $1332 \mathrm{keV}$.[4]

$$
\dot{D}(G y / \sec )=\frac{2 \cdot D(G y) \cdot A(B q)}{N}
$$

\section{Results and discussions}

With an Intel ${ }^{\circledR}$ Core TM i5 CPU $2.6 \mathrm{GHz}$ computer, we simulate by code GEANT4, the absorbed dose rates received by the whole body in four phantom positions inside the ionization cell; at 1, 1.5, 2 and $2.5 \mathrm{~m}$ from the center of the source cage of the gamma irradiator (see figure 2). All statistical errors are less than $2 \%$. The results are given in figure 3 .

The phantom body is exposed to gamma radiation from all directions (global exposure), Indeed gamma radiation can come directly from radioactive sources or can be reflected on the walls of the cell. As shown in figure 3 , by moving away from radioactive sources, the absorbed dose rate decreases, as well as the dangerousness of gamma radiation is also reduced, due to the increase in the air attenuation layer and the decrease in influence of gamma particles (the number of particles that arrive on the body per unit area). In addition to the body-source distance, the severity of gamma radiation strongly depends on exposure time, that is to say the residence time in front of the irradiator (see Fig.4).[11]

The longer the exposure time, the greater the dose absorbed by the human body and the more acute the effects of gamma radiation (deterministic effects). Depending on the intensity of the dose reached, these effects vary between nausea, vomiting, headache, rapid and severe drop in lymphocytes, serious digestive disorders up to shock and inevitable death (often for doses above 15 Gy). Depending on the organ or tissue affected, the duration of these symptoms varies from a few hours to a few weeks. Other types of effects can occur but in the long term are so-called stochastic effects such as cancer and other hereditary diseases, which appear years after exposure. The probability of occurrence of stochastic effects is proportional to the dose and they are more likely to appear for long-term low dose rate irradiation. $[12,13]$

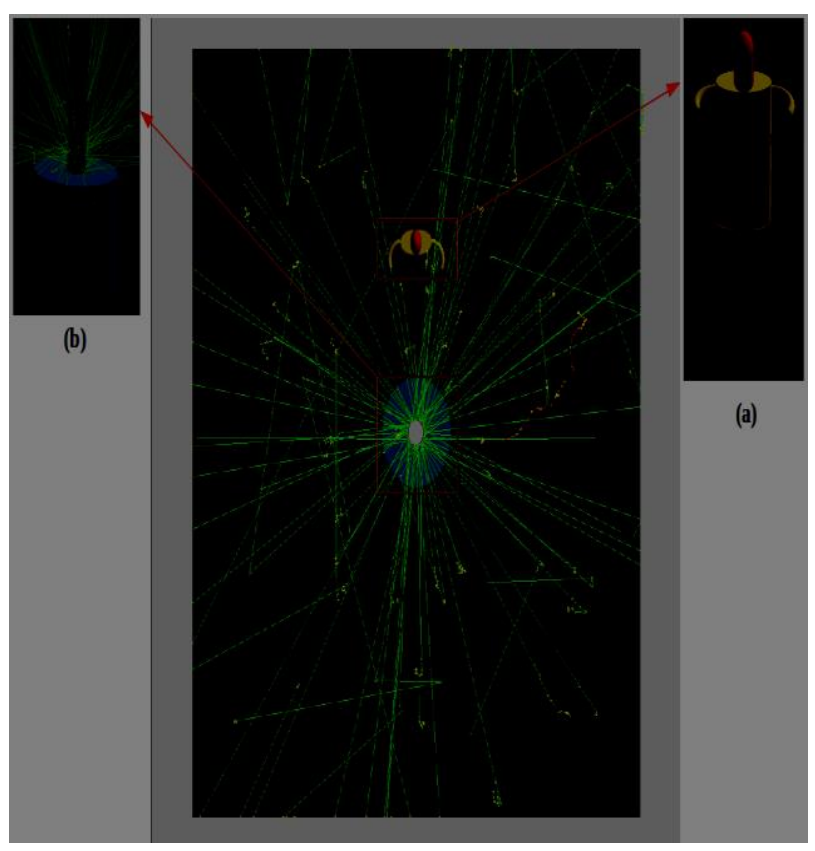

Fig. 2. Simulated view of the phantom during irradiation inside the ionization cell, (a) the anthropomorphic phantom (b) the gamma irradiator with its storage container. 
The absorbed dose also depends on the aging of the gamma irradiator, say on the activity of the source rods.

Figure 5 describes the variation in dose rate in the 4 positions as a function of the age of the source rods, starting with the date $07 / 2020$ until 07/2030.

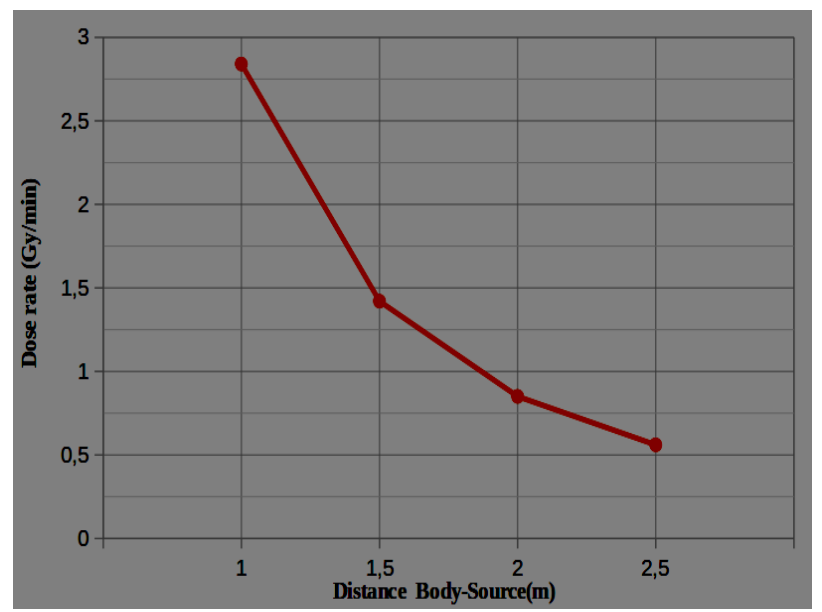

Fig. 3. Absorbed dose rates obtained by simulation in the 4 positions inside the ionization cell.

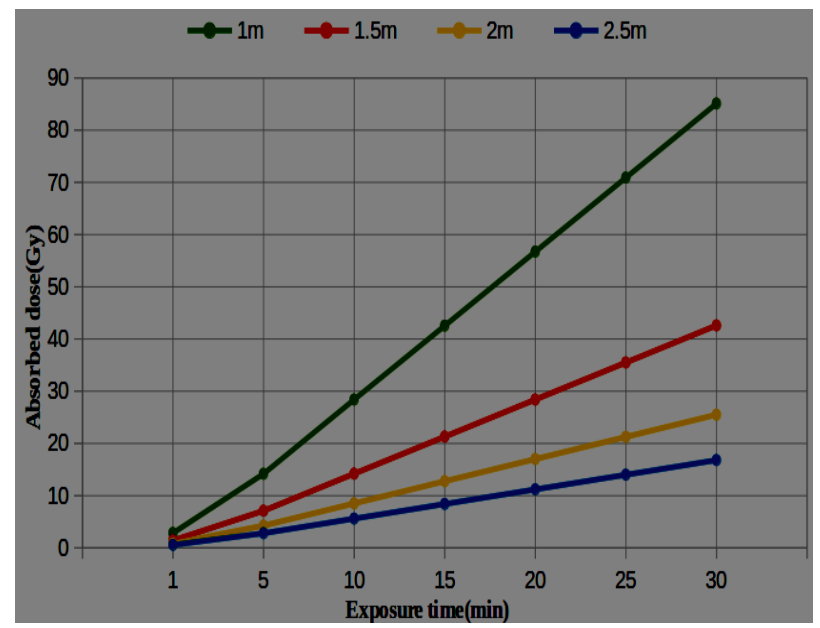

Fig. 4. Variation of the absorbed dose to the whole body as a function of exposure time and body-source distance.

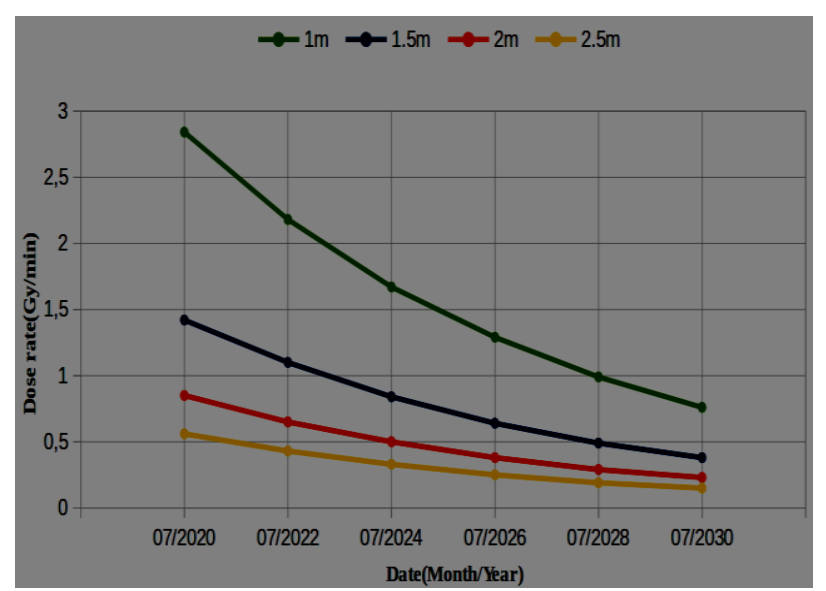

Fig. 5. Variation in absorbed dose rate as a function of the aging of the source rods

\section{Conclusion}

In this work, an assessment of the risks of exposure by gamma radiation comes from the NIAR cobalt-60 gamma irradiator was made. This evaluation is made by simulating through the Monte Carlo simulation, the dose absorbed by an anthropomorphic phantom assimilated to an adult man as a function of the exposure time, the phantom-source distance and the activity of the gamma irradiator. After this work, the workers and visitors of NIAR from different disciplines will be more aware and conscious of the risks of exposure by ionizing radiation such as gamma rays, and they will be more respectful of the rules of radiation protection and nuclear safety and security. The use of computational phantoms and monte carlo simulation for these types of studies has become essential and is a good alternative to experimental methods. Undoubtedly, this work will be a good initiative for other applications in the future at the ionization station of NIAR as for, the planning and management of radiological emergencies that may occur and others.

\section{References}

1. A. Aknouch, M. Mouhib, R. Sebihi, A. Didi, Y. Elouardi, A. Boubekraoui et A, Choukri, Mosc. Univ. Phys. Bull, vol. 75, No. 1, pp. 35-38 (2020)

2. National Research Council, Health Risksfrom Exposure to Low Levels of Ionizing Radiation: BEIR VII Phase 2, The National Academies Press, Washington, DC, 2006, p. 7.

3. R.E. Shore, et al., Implications of recent epidemiologic studies for the linear nonthreshold model and radiation protection, J. Radiol. Prot. 38 (2018) 1217e1233.

4. A. Aknouch, Y. El-ouardi, M. Mouhib, R. Sebihi, A. Didi, et A, Choukri, Mosc. Univ. Phys. Bull, (to be published)

5. Agostinelli, S. et al. Geant4 a simulation toolkit. Nucl. Instrum. Methods Phys. Res. ,2003.

6. Geant4 Collaboration. Introduction to Geant4:Release 10.5, Dec 2018.

7. Y. El-ouardi, A. Dadouch, A. Aknouch, M. Mouhib, A. Didi, Mosc. Univ. Phys. Bull, (to be published)

8. W.S. Snyder, et al, "MIRD Pamphlet No. 5 Revised, Estimates of absorbed fractions for monoenergetic photon sources uniformly distributed in various organs of a heterogeneous phantom",J. Nucl. Med. Suppl., no. 3, pp. 5-52, 1969.

9. V. Giacometti et al. Development of a high resolution voxelised head phantom for medical physics applications, Physica Medica(2017)

10. Habib Zaidi and Xie George $\mathrm{Xu}$, Computational Anthropomorphic Models of the Human Anatomy:The Path to Realistic Monte Carlo Modeling in Radiological Sciences, Annu. Rev. Biomed. Eng. 9:471-500(2007). 
11. ICRP Publication 60 (1990) Recommendations of the International Commission on Radiological Protection, Ann. ICRP 21(1-3).

12. Chander Mohan PATHAK et al., Whole Body Exposure to Low-dose Gamma Radiation Promotes Kidney Antioxidant Status in Balb/c Mice, J. Radiat. Res., 48, 113-120 (2007)

13. Roland Masse, Rayonnements ionisants, C.R. Acad. Sci. Paris, Sciences de la vie / Life Sciences 323 (2000) 633-640 - Elsevier 